\title{
Entanglement measure for the universal classes of fractons
}

\author{
Wellington da Cruz, S. Shelly Sharma \\ Departamento de Física, \\ Universidade Estadual de Londrina, Caixa Postal 6001, \\ Cep 86051-970 Londrina, PR, Brazil \\ E-mail address: wdacruz@exatas.uel.br and shelly@uel.br
}

(January 24, 2019)

\begin{abstract}
We introduce the notion of entanglement measure for the universal classes of fractons as an entanglement between ocuppation-numbers of fractons in the lowest Landau levels and the rest of the many-body system of particles. This definition came as an entropy of the probability distribution $\grave{a}$ la Shannon. Fractons are charge-flux systems classified in universal classes of particles or quasiparticles labelled by a fractal or Hausdorff dimension defined within the interval $1<h<2$ and associated with the fractal quantum curves of such objects. They carry rational or irrational values of spin and the spin-statistics connection takes place in this fractal approach to the fractional spin particles. We take into account the fractal von Neumann entropy associated with the fractal distribution function which each universal class of fractons satisfies. We consider the fractional quantum Hall effect-FQHE given that fractons can model Hall states. According to our formulation entanglement between occupaton-numbers in this context increases with the universality classes of the quantum Hall transitions considered as fractal sets of dual topological quantum numbers filling factors. We also introduce a relative entropy entanglement for fractons. We verify that the Hall states have stronger entanglement between ocuppation-numbers and so we can consider this resource for fracton quantum computing.
\end{abstract}

PACS numbers: 03.65.Ud, 03.65.Yz, 71.10.Pm, 05.30.Pr, 73.43.-f

Keywords: Entanglement, Quantum computation; Fractal von Neumann entropy; Fractons; Fractional quantum Hall effect. 


\section{INTRODUCTION}

Entanglement is a foundational characteristic of the quantum systems. This concept along of years has been considered in foundations of quantum theory and, more recently, as a physical resource for quantum information processing. There is a great effort today to formulate an entanglement theory just for extract the right answers of questions like how to measure, quantify and classify quantum correlations for implement quantum computation [1]. An entanglement state is a state which cannot be written as a direct product of states of the parts of a composite quantum system. Several entanglement measure are known, in particular, for bipartite system but no well defined for mixed multipartite systems. Hence a general definition is an open problem.

Now in strongly correlated systems as fractional quantum Hall effect-FQHE such ideas of entanglement and its quantification can give us some insight for understanding this type of macroscopic complex system. FQHE presents subtle properties of entanglement for different Hall states and in the literature is suggested that the theory of entanglement of quantum computing is a suitable tool for this investigation. The relevance of this perspective, for example, to a deeper understanding of quantum phase transitions, has been emphasized by leading researches. In [2] entanglement properties of the Laughlin wave functions for filling factors ( $f=1 / m$, with $\mathrm{m}$ is an odd number ) and for wave functions generated by the K-matrix of quantum Hall liquid $(f=2 /(2 m+1))$ was studied in terms of an entanglement measure of indistinguishable fermions. Another entanglement measure in terms of ocuppation-numbers for fermions was discussed in [3].

In this Letter, we introduce a notion of entanglement measure for the universal classes of fractons. These objects are charge-flux systems which carry rational or irrational values of spin and are classified in universal classes of particles or quasiparticles labelled by a fractal or Hausdorff dimension defined into the interval $1<h<2$. The spin of the particles are related with the Hausdorff dimension by $h=2-2 s, 0<s<\frac{1}{2}$ and this expression is a physical analogous to the fractal dimension formula of the graph of the functions, in the context of the fractal geometry, and given by: $\Delta(\Gamma)=2-H$, where $H$ is known as Hölder exponent, with $0<H<1[4]$.

The bounds of the fractal dimension $1<\Delta(\Gamma)<2$ needs to be obeyed in order for a function to be a fractal, so the bounds of our parameter $h$ are defined such that, for $h=1$ we have fermions, for $h=2$ we have bosons and for $1<h<2$ we have fractons. The Hölder exponent characterizes irregular functions which appears in diverse physical systems [5]. The fractal character of the quantum paths was observed in the path integral approach of the quantum mechanics [6]. Thus the fractal dimension is associated with the fractal quantum curves of fractons. In another way the fractal properties of the quantum paths can be extracted from the propagators of the particles in the momentum space $[7,8]$ and so our expression relating $h$ and $s$ can once more be justified ( for another view, see below ). The physical formula introduced by us, when we consider the spin-statistics relation $\nu=2 s$, is written as $h=2-\nu, 0<\nu<1$. This way, a fractal spectrum was defined taking into account a mirror symmetry:

$$
\begin{aligned}
& h-1=1-\nu, \quad 0<\nu<1 ; \quad h-1=\nu-1, \quad 1<\nu<2 \text {; } \\
& h-1=3-\nu, \quad 2<\nu<3 ; \quad h-1=\nu-3, \quad 3<\nu<4 ; \text { etc. }
\end{aligned}
$$


The statistical weight for these classes of fractons is given by [7]

$$
\mathcal{W}[h, n]=\frac{[G+(n G-1)(h-1)] !}{[n G] ![G+(n G-1)(h-1)-n G] !}
$$

and from the condition of the entropy be a maximum, we obtain the fractal distribution function

$$
n[h]=\frac{1}{\mathcal{Y}[\xi]-h}
$$

The function $\mathcal{Y}[\xi]$ satisfies the equation

$$
\xi=\{\mathcal{Y}[\xi]-1\}^{h-1}\{\mathcal{Y}[\xi]-2\}^{2-h}
$$

with $\xi=\exp \{(\epsilon-\mu) / K T\}$. The statistical weight can be written in terms of gamma function

$$
\begin{aligned}
\mathcal{W}[x, y] & =\frac{\Gamma(x+y+1)}{\Gamma(x+1) \Gamma(y+1)} \\
& =\frac{(x+y) !}{x ! y !} \\
& =\mathcal{W}[y, x]
\end{aligned}
$$

where $x[h]=N=n G$ and $y[h]=G+(N-1) h+1-2 N$, such that for bosons $y[h=2]=G-1$ and fermions $y[h=1]=G-N$. For particles with spin defined into the interval $0 \leq s \leq \frac{1}{2}$, we obtain $y[s]=G-(N-1) 2 s-1$, so in the large $N$ limit we have $h=2-2 s$ and for the statistical parameter within the interval $0 \leq \nu \leq 1$, we obtain $y[\nu]=G-(N-1) \nu-1$, and again in the large $N$ limit $h=2-\nu$, so $\nu=2 s$, i.e. the spin-statistics connection is established. We can check that all the interpolating expressions have the same bounds. Each expression reduces to the other one.

We understand the fractal distribution function as a quantum-geometrical description of the statistical laws of nature, since the quantum path is a fractal curve ( a point noted by Feynman ) and this reflects the Heisenberg uncertainty principle. The Eq.(3) embodies nicely this subtle information about the quantum paths associated with the particles.

We can obtain for any class its distribution function considering the Eqs.(3,4). For example, the universal class $h=\frac{3}{2}$ with distinct values of spin $\left\{\frac{1}{4}, \frac{3}{4}, \frac{5}{4}, \cdots\right\}_{h=\frac{3}{2}}$, has a specific fractal distribution

$$
n\left[\frac{3}{2}\right]=\frac{1}{\sqrt{\frac{1}{4}+\xi^{2}}} .
$$

This result coincides with another one of the literature of fractional spin particles for the statistical parameter $\nu=\frac{1}{2}$ [9], however our interpretation is completely distinct. This particular example, shows us that the fractal distribution is the same for all the particles into the universal class labelled by $h$ and with different values of spin ( consider the fractal 
spectrum, for a simple check ). Thus, we emphasize that in our formulation the spinstatistics connection is valid for such fractons. The authors in [9] ignored this possibility. Therefore, our results give another perspective for the fractional spin particles or anyons [10]. By the way, we can obtain straightforward the Hausdorff dimension associated to the quantum paths of the particles with any value of spin. This constitutes a fine result of our approach.

We also have

$$
\xi^{-1}=\{\Theta[\mathcal{Y}]\}^{h-2}-\{\Theta[\mathcal{Y}]\}^{h-1}
$$

where

$$
\Theta[\mathcal{Y}]=\frac{\mathcal{Y}[\xi]-2}{\mathcal{Y}[\xi]-1}
$$

is the single-particle partition function. We verify that the classes $h$ satisfy a duality symmetry defined by $\tilde{h}=3-h$. So, fermions and bosons come as dual particles. As a consequence, we extract a fractal supersymmetry which defines pairs of particles $\left(s, s+\frac{1}{2}\right)$. This way, the fractal distribution function appears as a natural generalization of the fermionic and bosonic distributions for particles with braiding properties. Therefore, our approach is a unified formulation in terms of the statistics which each universal class of particles satisfies, from a unique expression we can take out any distribution function.

The fractal von Neumann entropy per state in terms of the average occupation number is given as [7]

$$
\mathcal{S}_{G}[h, n]=K\left[[1+(h-1) n] \ln \left\{\frac{1+(h-1) n}{n}\right\}-[1+(h-2) n] \ln \left\{\frac{1+(h-2) n}{n}\right\}\right]
$$

and it is associated with the fractal distribution function Eq.(3).

Now taking into account this entropy, we define an entanglement measure for the universal classes of fractons in terms of the probability distribution $p$ as:

$$
\mathcal{E}[h, p]=[1+(h-1) p] \log _{2}\left\{\frac{1+(h-1) p}{p}\right\}-[1+(h-2) p] \log _{2}\left\{\frac{1+(h-2) p}{p}\right\},
$$

with $0 \leq p \leq 1$, where $p$ is the probability of a state above the lowest Landau levels-LLL be empty, partialy filled or completly filled. Of course, we can consider $p=p(n)$, with $n$-states above the LLL, such that:

$$
\begin{aligned}
\mathcal{E}[h, p]= & \sum_{n}\left[[1+(h-1) p(n)] \log _{2}\left\{\frac{1+(h-1) p(n)}{p(n)}\right\}\right. \\
& \left.-[1+(h-2) p(n)] \log _{2}\left\{\frac{1+(h-2) p(n)}{p(n)}\right\}\right] .
\end{aligned}
$$

Thus $\mathcal{E}[h, p]$ measures the entanglement between ocuppation-numbers of fractons considered in the LLL and the rest of the system. The Eq.(12) for fermions reduces to [3]

$$
\mathcal{E}[h=1, p]=-p \log _{2} p-(1-p) \log _{2}(1-p) .
$$


In another way, for the index $n$ we can consider distinct probability distribution $q(n)$ and $p(n)$, so we define a relative entanglement entropy as:

$$
\begin{aligned}
\mathcal{E}_{\mathcal{R}}[h, p \| q]= & \sum_{n}\left[[1+(h-1) p(n)] \log _{2}\left\{\frac{p(n)[1+(h-1) p(n)]}{q(n)}\right\}\right. \\
& \left.+[1+(h-2) p(n)] \log _{2}\left\{\frac{q(n)[1+(h-2) p(n)]}{p(n)}\right\}\right],
\end{aligned}
$$

and for fermions we obtain [11]

$$
\begin{aligned}
\mathcal{E}_{\mathcal{R}}[h=1, p \| q] & =p \log _{2} p+(1-p) \log _{2}(1-p)-p \log _{2} q \\
& =-\mathcal{E}[h=1, p]-p \log _{2} q .
\end{aligned}
$$

\section{ENTANGLEMENT IN THE FQHE}

In [7] we have considered a fractal approach to the FQHE [12] with Hall states modeled by fractons. According to our formulation the quantum Hall states associated with a specific filling factor is a fracton state with value of $\operatorname{spin} s=\nu / 2$. The filling factors which characterize the quantization of the Hall resistance, have the same value of the statistical parameter, i.e. $\nu=f$ (numerically), where $f$ is defined by $f=N \frac{\phi_{0}}{\phi}$, and $N$ is the electron number, $\phi_{0}$ is the quantum unit of flux and $\phi$ is the flux of the external magnetic field throughout the sample. The spin-statistics relation is given by $\nu=2 s=2 \frac{\phi \prime}{\phi_{0}}$, where $\phi$ ' is the flux associated with the charge-flux system which defines the fracton $(h, \nu)$. In this way the universality classes of the quantum Hall transitions satisfy some properties of a subgroup of the modular group $S L(2, \mathbf{Z})$ related with the Farey sequences of rational numbers. The transitions allowed are those generated by the condition $\left|p_{2} q_{1}-p_{1} q_{2}\right|=1$, with $\nu_{1}=\frac{p_{1}}{q_{1}}$ and

$\nu_{2}=\frac{p_{2}}{q_{2}}[13,7]$. This way we define the universality classes of the quantum Hall transitions in terms of fractal sets labelled by the Hausdorff dimension. We verify that the filling factors experimentally observed appear into the classes $h$ and from the definition of duality between the fractal sets, we note that the FQHE occurs in pairs of dual filling factors. These quantum numbers get their topological character from the fractal dimension associated with the quantum paths. Our results show clearly which the FQHE has a fractal-like structure and this deeper feature is revealed by robust mathematical concepts. Another fractal formulation to the FQHE was discussed in [14] and considered as an approach to be explored for understanding the subtle properties of the FQHE [15]. Here we observe that our program antecipated this suggestion just considering properly ideas of the fractal geometry [7].

The entanglement properties of fractons ( Hall states ) can be now analysed considering the Eq.(12). For distinct classes of fractons we have verified that $\mathcal{E}[h, p] \neq \mathcal{E}[h, 1-p]$ in contrast with $\mathcal{E}[h=1, p]=\mathcal{E}[h=1,1-p]$ for fermions. The entanglement measure is a concave function with maximum for state partialy filled, with $\mathcal{E}[h, p=0]=0$ and $\mathcal{E}[h, p=1] \neq 0$ and this signalizes which for states completly filled there exists yet a residual entanglement between fractons in the LLL and the rest of the system. The entanglement increases when we run in the interval $1<h<2$, for instance, $\mathcal{E}[h=4 / 3]<\mathcal{E}[h=3 / 2]<$ $\mathcal{E}[h=5 / 3]$. We have, 


$$
\cdots \rightarrow\left\{\frac{2}{3}, \frac{4}{3}, \cdots\right\}_{h=\frac{4}{3}} \rightarrow\left\{\frac{1}{2}, \frac{3}{2}, \cdots\right\}_{h=\frac{3}{2}} \rightarrow\left\{\frac{1}{3}, \frac{5}{3}, \cdots\right\}_{h=\frac{5}{3}} \rightarrow \cdots
$$

such that for the entanglement measure written in terms of the filling factors

$$
\mathcal{E}[\nu, p]=[1+(1-\nu) p] \log _{2}\left\{\frac{1+(1-\nu) p}{p}\right\}-[1-\nu p] \log _{2}\left\{\frac{1-\nu p}{p}\right\},
$$

with $0<\nu<1$, and we confirme that $\mathcal{E}[\nu=2 / 3]<\mathcal{E}[\nu=1 / 2]<\mathcal{E}[\nu=1 / 3]$. For the other members of the classes we need consider the fractal spectrum Eq.(1)

$$
\mathcal{E}[\nu, p]=[1+(\nu-1) p] \log _{2}\left\{\frac{1+(\nu-1) p}{p}\right\}-[1+(\nu-2) p] \log _{2}\left\{\frac{1+(\nu-2) p}{p}\right\}
$$

with $1<\nu<2$; etc. So we have $\mathcal{E}[\nu=4 / 3]<\mathcal{E}[\nu=3 / 2]<\mathcal{E}[\nu=5 / 3]$. This way we verify that the Eq.(12) for the class $h$, is the same for all the members of the class. Observe that our approach gives information about the entanglement for any possible wave function associated with a specific value of the filling factor. In another route we can consider the LLL for fractons, i.e. if the temperature is sufficiently low and $\epsilon<\mu$, we can check that the mean ocuppation number Eq.(3) is given by $n=\frac{1}{2-h}$, and so the fractal parameter $h$ regulates the number of particles in each quantum state. For $h=1, n=1 ; h=2, n=\infty$; etc. At $T=0$ and $\epsilon>\mu, n=0$ if $\epsilon>\epsilon_{F}$ and $n=\frac{1}{2-h}$ if $\epsilon<\epsilon_{F}$, hence we get a step distribution, taking into account the Fermi energy $\epsilon_{F}$ and $h \neq 2$. We can check that for $h=\frac{4}{3}, \frac{3}{2}, \frac{5}{3}$ we obtain $n=\frac{3}{2}, \frac{2}{1}, \frac{3}{1}$, respectively. In the first case we have three particles for two states, in the second case two particles for one state and in the last case three particles for one state. So when we run in the interval $1<h<2$ we gain more particles for each possible state. In some sense fractons can be understood as quasifermions when near the universal class $h=1$ and as quasibosons when near the universal class $h=2$. The entanglement of the FQHE increases because we have more particles ( fractons ) and less states. On the other hand, in terms of the filling factors, the average ocuppation number can be written as $n=\frac{1}{\nu}$, $0<\nu<1 ; n=\frac{1}{2-\nu}, 1<\nu<2$; etc. We obtain the pairs $\left(\nu=\frac{2}{3}, n=\frac{3}{2}\right) ; \quad\left(\nu=\frac{1}{2}, n=\frac{2}{1}\right)$; $\left(\nu=\frac{1}{3}, n=\frac{3}{1}\right) ;\left(\nu=\frac{4}{3}, n=\frac{3}{2}\right) ;\left(\nu=\frac{3}{2}, n=\frac{2}{1}\right) ;\left(\nu=\frac{5}{3}, n=\frac{3}{1}\right) ;$ etc. The behaviour of the step distribution confirmes our former analysis: the ground state of the FQHE is a stronger entangled state and the entanglement between ocuppation-numbers of fractons in the LLL and the rest of the system shows us quantum correlations of equal amount. Also, we know how much they are.

All these results agrees with the entanglement properties of the Laughlin wave function and those generated by the K-matrix [2]. On the other hand, the FQHE understood in terms of the composite fermions or composite bosons are non-entangled as observed in [3], so in contrast, fractons appear as a suitable system for study the quantum correlations properties of the FQHE. Thus the suggestion that ideas of the quantum information science can give insights for understanding some complex quantum systems [16] is manifested in our definition of entanglement measure for the universal classes of fractons. The universality classes of the quantum Hall transitions as fractal sets of dual topological quantum numbers filling factors, according to our formulation, have increasing entanglement into the interval $1<h<2$ and this suggests fracton qubits as a physical resource for quantum computing. In 
the literature FQHE qubits associated with the geometrical characteristic of the fractional spin particles have been exploited [17].

The relative entanglement entropy for fractons has the behaviour $\mathcal{E}_{\mathcal{R}}[h, p=q=0]=0$ and $\mathcal{E}_{\mathcal{R}}[h, p=q=1] \neq 0$, with $\mathcal{E}[h, p=1]>\mathcal{E}_{\mathcal{R}}[h, p=q=1]$. For fermions we have $\mathcal{E}_{\mathcal{R}}[h=1, q=p]=0$.

\section{CONCLUSIONS}

The discussion of the FQHE in terms of fractons shows us the potential application of this physical support for the implementation of quantum computing. The topological character of these objects is crucial against problems of the decoherence. Entangled fracton states can be considered as a stable resource for a topological quantum computation, i.e., a fault-tolerant quantum computation. We observe again that fractons carry values rational or irrational of spin ( magnetic moment ) which can be considered in experiments of NMR. In the literature geometric phases and the concept of anyon have been explored for implement quantum gates because they are robust against random noise of the environment [18].

Finally, we have introduced the notion of entanglement measure for the universal classes of fractons, where concepts of the fractal geometry appear naturally and gives us the opportunity of extract information about the quantum correlations of the ground state of the FQHE. Our approach reveals us the fractal nature of this phenomenon [7]. The possibility of establish a bridge between quantum information theory and the quantum Hall transitions go to the direction of some ideas of research in the literature and considered of extreme importance [16]. As we saw, our results agree with other one [2] and open an avenue for we speculate on a fracton quantum computing. 


\section{REFERENCES}

[1] J. Preskill, Lectures notes on quantum informatiom and computation, www.theory.caltech.edu/people/preskill/ph229;

M. Keyl, Phys. Rep. 369, 431 (2002);

D. $\operatorname{Bru} \beta$, J. Math. Phys. 43, 4237 (2002);

C. Brukner et al., quant-ph/0106119;

V. Vedral, quant-ph/0102094;

R. F. Werner, quant-ph/0101061;

B. M. Terhal, J. of Theor. Computer Science 287, 313 (2002);

M. Horodecki et al., quant-ph/0109124;

G. Ghirardi et al., quant-ph/0109017;

M. J. Donald et al., J. Math. Phys. 43, 4252 (2002);

A. Ekert et al., quant-ph/0011013;

M. Lewenstein et al., J. of M. Optics 47, 2841 (1998);

B. Schumacher and M. D. Westmoreland, quant-ph/0004045;

V. P. Belavkin and M. Ohya, quant-ph/9812082;

M. B. Plenio and V. Vedral, Contemp. Phys. 39, 431 (1998);

A. Ekert, quant-ph/9803072;

N. J. Cerf and C. Adami, Physica D 120, 62 (1998).

[2] B. Zeng et al., Phys. Rev. A 66, 042324 (2002).

[3] Y. Shi, quant-ph/0204058; Phys. Rev. A 67, 024301 (2003).

[4] B. B. Mandelbrot, The Fractal Geometry of Nature (Freeman, New York, 1982);

C. Tricot, Curves and Fractal Dimension (Springer-Verlag, New York,1995);

K. Falkoner, Fractal Geometry (Wiley, New York, 1990);

K. Falkoner, Techniques in Fractal Geometry (Wiley, New York, 1997);

K. Falkoner, The Geometry of Fractal Sets

(Cambridge University Press, Cambridge, 1985).

[5] K. M. Kolwankar and A. D. Gangal, Pramana J. Phys. 48, 49 (1997);

Chaos 6, 505 (1996);

P. Meakin, Fractals, Scaling and Growth far from Equilibrium

(Cambridge University Press, Cambridge,1998).

[6] R. P. Feynman and A. R. Hibbs, Quantum Mechanics and Path Integrals

( MacGraw-Hill, New York, 1965 ), pp. 176-177.

[7] W. da Cruz, Int. J. Mod. Phys. A 15, 3805 (2000),

W. da Cruz, Physica A 313, 446 (2002). See also:

W. da Cruz, Chaos, Solitons and Fractals 17, 975 (2003),

W. da Cruz, Int. J. Mod. Phys. A 18, 2213 (2003),

W. da Cruz, math-ph/0306071,

W. da Cruz, cond-mat/0304398,

W. da Cruz, cond-mat/0301587,

W. da Cruz, J. Phys: Cond. Matter. 12, L673 (2000),

W. da Cruz and R. de Oliveira, Mod. Phys. Lett. A 15, 1931 (2000),

W. da Cruz, Mod. Phys. Lett. A 14, 1933 (1999).

[8] A. M. Polyakov, in Proc. Les Houches Summer School vol. IL, ed. E. Brézin and J. Zinn-Justin (North Holland, 1990), p.305. 
[9] F. D. Haldane, Phys. Rev. Lett. 67, 937 (1991);

Y. S. Wu, Phys. Rev. Lett. 73, 922 (1994);

S. B. Isakov, Mod. Phys. Lett. B 8, 319 (1994);

A. K. Rajagopal, Phys. Rev. Lett. 74, 1048 (1995);

A. D. de Veigy and S. Ouvry, Phys. Rev. Lett. 72, 600 (1994).

[10] Comtet et al., (Eds.) Topological Aspects of Low Dimensional Systems, Les Houches 1998, vol. LXIX ( Springer Verlag, New York, 1999 );

A. Khare, Fractional Statistics and Quantum Theory ( World Scientific, Singapore, 1998 );

A. Lerda, Anyons: Quantum Mechanics of Particles with Fractional Statistics (Springer Verlag, Heidelberg, 1995);

J. M. Leinaas, Rep. Phys. 242, 371 (1994);

G. Dunne, Self-dual Chern-Simons Theories

(Springer Verlag, Heidelberg,1992);

S. Forte, Rev. Mod. Phys. 64, 193 (1992);

R. Iengo andK. Lechner, Rep. Phys. 213, 179 (1992);

F. Wilczek, Fractional Statistics and Anyon Superconductivity

( World Scientific, Singapore, 1990 );

F. Wilczek and Shapere, Geometric Phases in Physics

( World Scientific, Singapore, 1989 ); and references therein.

[11] M. A. Nielsen and I. L. Chung, Quantum Computation and Quantum Information

( Cambridge University Press, Cambridge, 2000 ), p. 504.

[12] R. B. Laughlin, Rev. Mod. Phys. 71, 863 (1999);

H. Stormer, Rev. Mod. Phys. 71, 875 (1999);

D. C. Tsui, Rev. Mod. Phys. 71, 891 (1999);

and references therein.

[13] B. P. Dolan, J. Phys. A 32, L243 (1999); Nucl. Phys. B554, 487 (1999);

C. P. Burgess and C. A. Lütken, Nucl. Phys. B 500, 367 (1997);

and references therein.

[14] R. G. Mani and K. von Klitzing, Z. Phys. B 100, 635 (1996).

[15] J. H. Smet, Nature 422, 391 (2003).

[16] M. A. Nielsen, quant-ph/0210005, quant-ph/0208078;

A. Osterloh et al., Nature 466, 608 (2002);

T. J. Osborne and M. A. Nielsen, quant-ph/0202162, quant-ph/0109024;

J. Preskil, J. Mod. Opt. 47, 127 (2000);

D. P. DiVicenzo, Fords. der Physik, 48, 771 (2000);

D. Aharonov, Phys. Rev. A 62, 2311 (1999);

and references therein.

[17] S. R. Eric Yang et al., Phys. Rev. B 66, 153302 (2002);

D. V. Averin and V. J. Goldman, cond-mat/0110193.

[18] V. Vedral, quant-ph/0212133;

P. Zanardi and M. Rasetti, Phys. Lett. A 264, 94 (1999);

J. Pachos, P. Zanardi and M. Rasetti, Phys. Rev. A 61, 010305(R) (2000);

J. Pachos and P. Zanardi, quant-ph/0007110;

A. Yu. Kitaev, quant-ph/9707021; 
R. Walter Ogburn and J. Preskill, Lectures notes in Computer Science, 1509, pp. 341356 (1999);

M. H. Freedman et al., Bulletin of AMS, 40, 31 (2002);

M. H. Freedman et al., Comm. Math. Phys. 227, 587 (2002);

S. Lloyd, quant-ph/0004010;

P. Zanardi and S. Lloyd, Phys. Rev. Lett. 90, 067902-1 (2003);

B. J. Overbosh and F. A. Bais, Phys. Rev. A 64, 062107(2001);

C. Mochon, quant-ph/0306063; Phys. Rev. A 67, 022315 (2003);

and references therein. 